\title{
Particle-hole symmetry breaking in the pseudogap state of Bi2201
}

\author{
Makoto Hashimoto ${ }^{1,2,3 \dagger}$, Rui-Hua He ${ }^{1,2 \uparrow}$, Kiyohisa Tanaka $a^{1,2,3,4}$, Jean-Pierre Testaud ${ }^{1,2,3}$, \\ Worawat Meevasana ${ }^{1,2}$, Rob G. Moore ${ }^{1,2}$, Donghui Lu ${ }^{1,2}$, Hong Yao', Yoshiyuki Yoshida ${ }^{5}$, \\ Hiroshi Eisaki ${ }^{5}$, Thomas P. Devereaux ${ }^{1,2}$, Zahid Hussain ${ }^{3}$ and Zhi-Xun Shen ${ }^{1,2 \star}$
}

In conventional superconductors, a gap exists in the energy absorption spectrum only below the transition temperature $\left(T_{c}\right)$, corresponding to the price to pay in energy for breaking a Cooper pair of electrons and creating two excited states. In high- $T_{c}$ cuprate superconductors above $T_{c}$ but below a temperature $T^{*}$, an energy gap called the pseudogap ${ }^{1}$ exists, and is controversially attributed either to pre-formed superconducting pairs, which would show particle-hole symmetry, or to competing phases that would typically break it. Scanning tunnelling microscopy (STM) studies suggest that the pseudogap stems from lattice translational symmetry breaking ${ }^{2-9}$ and is associated with a different characteristic spectrum for adding or removing electrons (particle-hole asymmetry; refs 2,3 ). However, no signature of either energy or spatial symmetry breaking of the pseudogap has previously been observed by angle-resolved photoemission spectroscopy ${ }^{9-18}$ (ARPES). Here we report ARPES data from Bi2201, which reveal both particle-hole symmetry breaking and pronounced spectral broadening-indicative of spatial symmetry breaking without long-range order at the opening of the pseudogap. Our finding supports the STM proposal that the pseudogap state is a broken-symmetry state that is distinct from homogeneous superconductivity.

The nature of the pseudogap can be explored by examining the dispersion of the occupied electronic states measured by ARPES. As shown in Fig. 1m, when a particle-hole symmetric gap opens from the normal-state dispersion (red curve) because of homogeneous superconductivity, one always expects an alignment between the Fermi momentum $k_{\mathrm{F}}$ and the 'back-bending' or saturation momentum (green arrows) of the dispersion in the gapped states (weighted blue curve). As a result of this strong constraint, the observation of a back-bending or dispersion saturation anomaly away from $k_{\mathrm{F}}$ in a gapped state can be conclusive evidence of a broken particle-hole symmetry nature of the gap, even though the information about the unoccupied state is absent. Here we note that the dispersion of the spectral peak position, regardless of the spectral weight, is a simple and direct way to address the issue of particle-hole symmetry. Owing to suppressed spectral weight that makes it hard to discern from the experimental background, the back-bending may sometimes be subtle and show up as a dispersion saturation. Nevertheless, it can be distinguished from a smooth dispersion where no dispersion saturation occurs.
Figure 1 shows the temperature evolution across $T^{*}$ of the Fermi-Dirac-function-divided ARPES spectra of a nearly optimally doped cuprate superconductor $\mathrm{Pb}_{0.55} \mathrm{Bi}_{1.5} \mathrm{Sr}_{1.6} \mathrm{La}_{0.4} \mathrm{CuO}_{6+\delta}$ $(\mathrm{Pb}-\mathrm{Bi} 2201)$ with $T_{\mathrm{c}} \sim 34 \mathrm{~K}$. Data were taken in an antinodal cut approximately along $(\pi,-\pi)-(\pi, 0)-(\pi, \pi)$, as shown in the inset of Fig. 1g. The spectra in the true normal state above $T^{*}$ $(\sim 125 \mathrm{~K})$ present a parabolic dispersion of the intensity maximum as a function of momentum with two clear Fermi level crossings at $k_{\mathrm{F}}$ momenta (red spectra) and a bottom reaching $E_{\mathrm{Bot}} \sim-20 \mathrm{meV}$ at $(\pi, 0)$ (Fig. 1a,g,n). Given that the data were taken at $160 \mathrm{~K}$, the measured spectra in the true normal state are remarkably simple, similar to that of ordinary metal ${ }^{19}$. Whereas this is naturally expected for a band in the absence of a gap, with the pseudogap opening around $E_{\mathrm{F}}$ below $T^{*}$, the spectra become surprisingly incoherent and the spectral weight centroid is transferred towards a higher binding energy (Fig. 1a-l). Despite the broadness of the spectra at low temperatures, the intensity maximum of each spectrum can be defined and traced as a function of momentum. The dispersion thus extracted becomes stronger towards lower temperatures with the band bottom at $(\pi, 0)$ being pushed far away from the true normal state $E_{\text {Bot }}$, as summarized in Fig. 1 . Well below $T^{*}$ where the spectra are fully gapped in the measured antinodal cut, no dispersion saturation or back-bending is observed at $k_{\mathrm{F}}$, defined by the Fermi crossing at $T=160 \mathrm{~K}$ (guides to the eyes in red). Instead, while approaching $E_{\mathrm{F}}$, the dispersion flattens and appears to bend back at momenta (green arrows) markedly away from $k_{\mathrm{F}}$. This misalignment can also be seen in the summary of the data in Fig. 1i-1 (the red spectra are for $k_{\mathrm{F}}$ and the green spectra are for possible back-bending momenta). In contrast to what is shown in Fig. $1 \mathrm{~m}$, the behaviour below $T^{*}$ is completely different from the expectation for the homogeneous superconducting state, suggesting that the transition from the true normal state above $T^{*}$ to the pseudogap state has a different origin. In addition to the misalignment between $k_{\mathrm{F}}$ and the back-bending momenta for $T \ll T^{*}$, the observed widening of the energy separation between the band bottom and the back-bending on decreasing temperature also contradicts the expectation in Fig. $1 \mathrm{~m}$ where the energy separation (or the overall dispersion) decreases with an opening of a superconducting gap.

The symmetry-breaking dispersion begins at the pseudogap opening temperature $T^{*} \sim 125 \pm 10 \mathrm{~K}$ determined by various independent measurements (ARPES; refs 9,10, resistivity ${ }^{20}$, NMR;

\footnotetext{
${ }^{1}$ Stanford Institute for Materials and Energy Sciences, SLAC National Accelerator Laboratory, 2575 Sand Hill Road, Menlo Park, California 94025, USA, ${ }^{2}$ Geballe Laboratory for Advanced Materials, Departments of Physics and Applied Physics, Stanford University, California 94305, USA, ${ }^{3}$ Advanced Light Source, Lawrence Berkeley National Lab, Berkeley, California 94720, USA, ${ }^{4}$ Department of Physics, Osaka University, Toyonaka, Osaka 560-0043, Japan, ${ }^{5}$ Nanoelectronics Research Institute, Advanced Industrial Science and Technology, Tsukuba, Ibaraki 305-8568, Japan. ${ }^{\dagger}$ These authors contributed equally to this work. *Present address: School of Physics, Suranaree University of Technology, Nakhon Ratchasima, 30000 Thailand. *e-mail: zxshen@stanford.edu.
} 

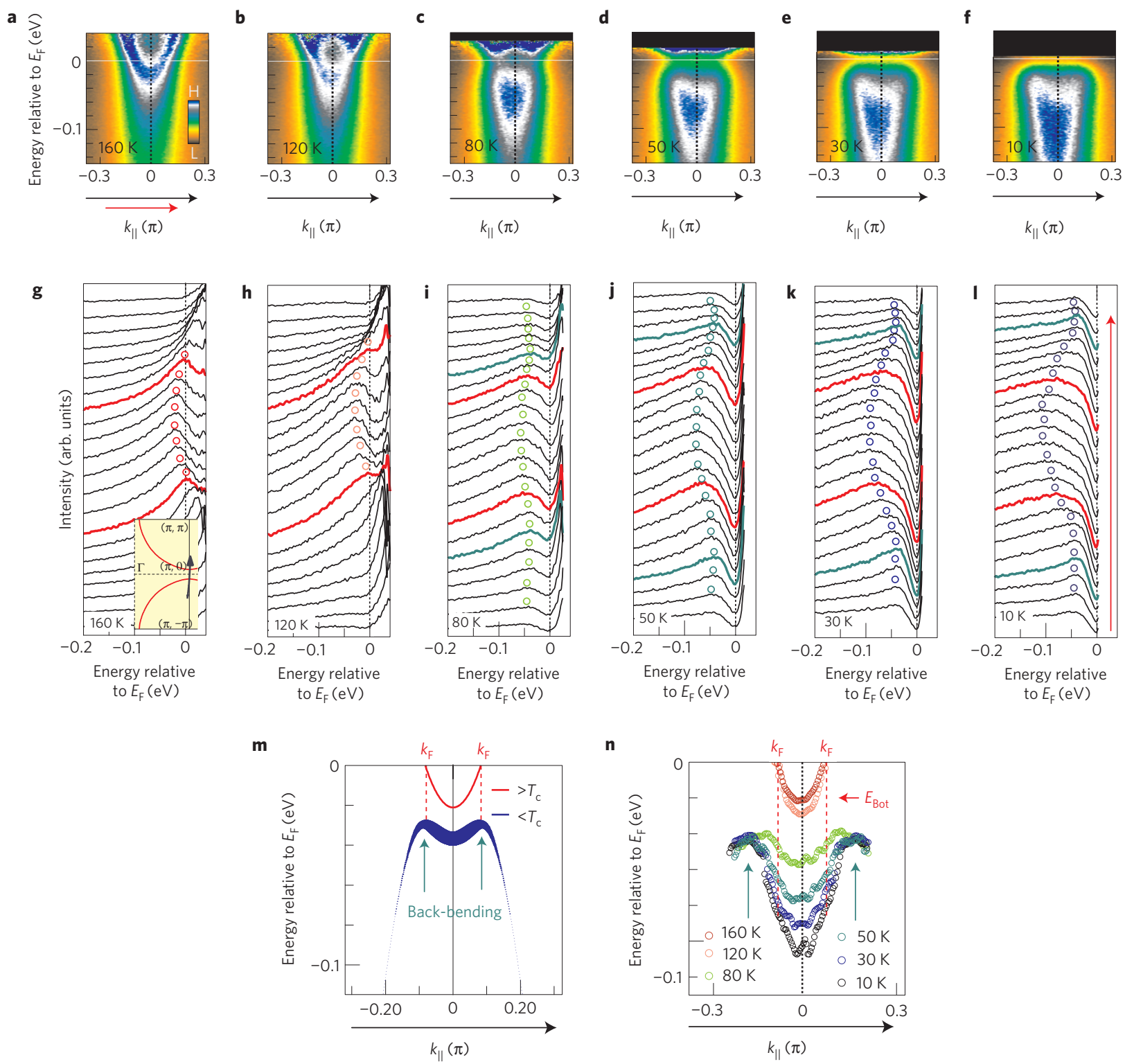

Figure 1 | Particle-hole symmetry breaking in the antinodal dispersion of pseudogapped Pb-Bi2201. $T_{\mathrm{C}}=34 \mathrm{~K}, \mathrm{~T}^{*}=125 \pm 10 \mathrm{~K}$.

$\mathbf{a}-\mathbf{I}$, Fermi-Dirac-function-divided image plots (a-f) and corresponding spectra as a function of parallel momentum ( $\mathbf{g}-\mathbf{I}$ ) taken along the antinodal cut shown in the inset of $\mathbf{g}$ at selected temperatures. The intensity maximum of each spectrum is marked by a circle. The typical error bars estimated in the analysis of the smoothed first-derivative spectra are smaller than the symbol size. An extra shoulder feature of weak dispersion is seen close to $E_{\mathrm{F}}$ at $10 \mathrm{~K}$. Note that this feature at low energy has been a main focus in previous studies ${ }^{9-18}$. The spectra in red and green are at $k_{\mathrm{F}}$ and possible back-bending momenta of the intensity maximum dispersion, respectively (Supplementary Figs S1 and S2). $\mathbf{m}$, Simulated dispersion for $d$-wave homogeneous superconductivity with order parameter $V=30 \mathrm{meV}$. The quasiparticle energy for a given momentum state $k$ is given by $E(k)=\sqrt{\varepsilon(k)^{2}+\Delta(k)^{2}}$. Cuts are along $(\pi,-\pi)-(\pi, 0)-(\pi, \pi)$. The red (blue) curve is for the true normal (gapped) state. The spectral weight is indicated by the curve thickness. The back-bending (or saturation) of the dispersion and $k_{\mathrm{F}}$ are indicated in the panel. Note that the back-bending momentum in the gapped state remains aligned with $k_{\mathrm{F}}$. See details in Supplementary Methods. $\mathbf{n}$, Summary of the intensity maximum dispersions at different temperatures. The typical error bars from the derivative analysis are smaller than the symbol size.

ref. 21) on Bi2201 near the optimal doping. This is examined from the temperature dependence of spectra at two representative momenta, $(\pi, 0)$ and $k_{\mathrm{F}}$ (Fig. 2a,b). With increasing temperature, the energy positions of the spectral intensity maxima continuously move towards lower binding energies until they saturate at $E_{\mathrm{Bot}}$ and $E_{\mathrm{F}}$, respectively, at $T^{*}$ (Fig. $2 \mathrm{c}$ ). The smooth temperature evolution on cooling down from the true normal state with a clear onset at $T^{*}$ suggests that the dispersion of the intensity maximum found at $T<T^{*}$ (Fig. $1 \mathrm{n}$ ) is directly related to the pseudogap physics. That is, the misalignment between $k_{\mathrm{F}}$ and the back-bending momenta at $T \ll T^{*}$ is due to the pseudogap opening. This provides compelling evidence for a violation of the momentum structure expected from a particle-hole symmetric homogeneous superconducting state below $T^{*}$. This finding goes beyond the earlier STM works that revealed the broken lattice symmetry nature of the low-temperature phase, but lacked the detailed temperature dependence linking such symmetry breaking to the pseudogap opening below $T^{*}$.

Besides the band dispersion, the spectral intensity also shows a close link to the pseudogap opening. In Fig. $2 \mathrm{~d}$ we plot the spectral intensity at $k_{\mathrm{F}}$ in the energy window of $[-0.36,-0.30] \mathrm{eV}$ as a function of temperature, with the spectra normalized either by the photon flux, which reflect the raw spectral weight, or by a selected 

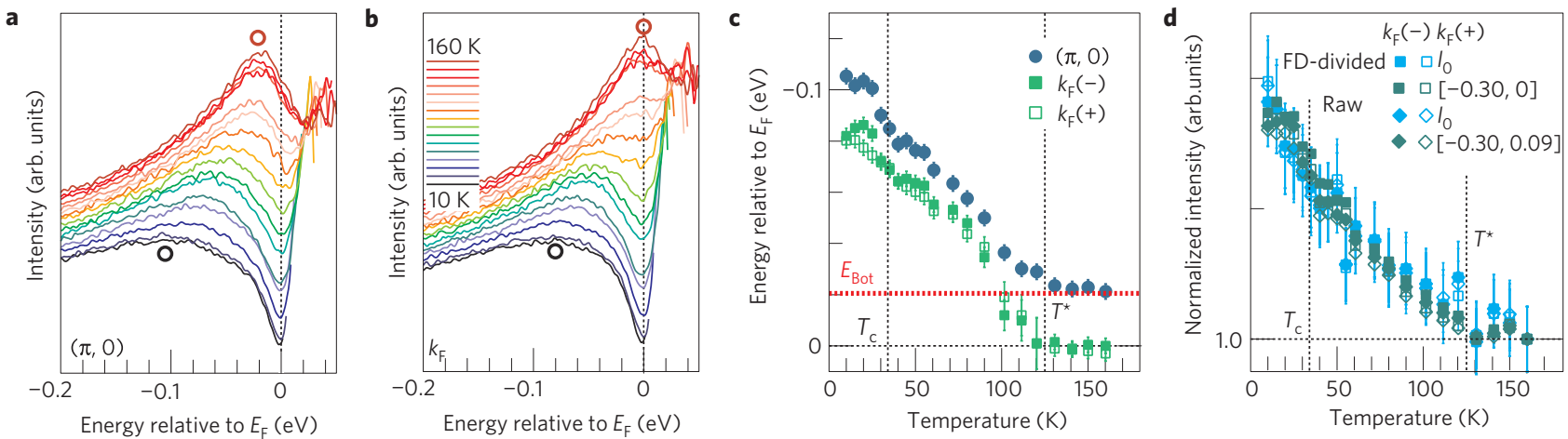

Figure 2 | Temperature evolution of the antinodal spectra tied to the pseudogap opening. a,b, Fermi-Dirac-divided spectra at ( $\pi, 0)(\mathbf{a})$ and $k_{F}(\mathbf{b})$, with a constant vertical offset between each spectrum after the normalization at the highest binding energy, taken at different temperatures as colour coded in increments of $10 \mathrm{~K}$. The intensity maxima at 160 and $10 \mathrm{~K}$ are marked by circles. Note that the low-energy shoulder feature loses its clear definition roughly above $T_{\mathrm{c}}$ on increasing the temperature. The offset and normalization are for better visualization of the spectral line shape and the intensity maximum feature but not for the discussion of the spectral weight redistribution (see Supplementary Fig. S3 for photon flux normalization).

c, Temperature-dependent energy position of the intensity maximum at $(\pi, 0)$ and two $k_{F}$ momenta along the antinodal cut. The error bars are estimated in the analysis of the smoothed first-derivative spectra. $\mathbf{d}$, Temperature dependence of the average intensity within $[-0.36,-0.30] \mathrm{eV}$ after spectral weight normalization by the photon flux $\left(I_{0}\right)$ and within selected energy ranges as denoted, of the raw and Fermi-Dirac (FD)-divided spectra at two $k_{F}$ momenta (see Supplementary Fig. S3 for the normalized spectra). The average intensity is normalized at $160 \mathrm{~K}$ for comparison with its typical error bars ( $\pm 5 \%$ for $I_{0}$ normalization and smaller than the symbol size for the area normalization). Note that any finite temperature dependence indicates spectral weight redistribution beyond the window.
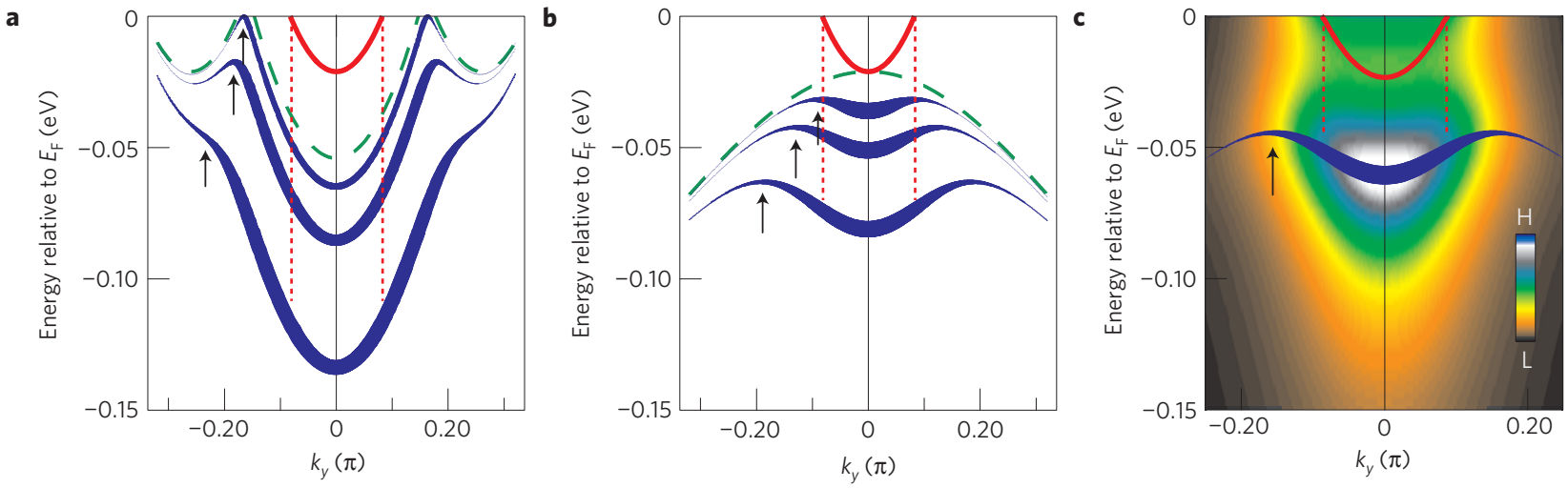

Figure 3 | Simulated spectra for simple options of the density-wave correlations behind the pseudogap. Cuts are along $(\pi,-\pi)-(\pi, 0)-(\pi, \pi)$ for all cases. a,b, Two long-range orders: incommensurate checkerboard density-wave order of orthogonal wavevectors $[0.26 \pi, 0]$ and $[0,0.26 \pi](\mathbf{a})$ and commensurate $[\pi, \pi]$ density-wave order $(\mathbf{b})$. The green dashed curves are shadow bands associated with the corresponding order, which interact with the bare band (red) producing the renormalized band dispersions (blue) presumably observable in the ordered or locally ordered state. The vertical red dashed lines are guides for the eyes for $k_{F}$. Selected renormalized band dispersions for the same eigenstate with order parameter $V=15,30$ and 60 meV (from top to bottom), independent of $k$, are shown for each case, to exemplify the misalignment between $k_{F}$ and the back-bending (or saddle point) momentum (arrow). The spectral weight is indicated by the curve thickness. Note that only first-order band folding is considered for simplicity in simulations for a. See Supplementary Fig. S7 for the complete results of all long-range density-wave simulations. $\mathbf{c}$, The $[\pi, \pi]$ density-wave order with finite correlation length: 10 lattice constants, and density-wave gap $\Delta_{k}=60 \mathrm{meV}$, independent of $k$. The extracted dispersion is shown by the blue curve, with its thickness proportional to the peak intensity. For $\mathbf{a}$ and $\mathbf{b}$, note that a pronounced upshift of the band bottom commonly requires a decreasing $V$, which inevitably results in corresponding changes of the back-bending position, in contrast to the 'pinning' of it possibly observed below $\sim 50 \mathrm{~K}$ (Fig. 1n). This could be consistent with the reported temperature independence of back-bending momenta across $T_{c}$ (ref. 12), and poses a challenge for the exclusive density-wave picture put in such simple ways. See details in Supplementary Methods.

energy window as indicated in the figure (see Supplementary Fig. S3 for the spectra with these normalizations). Regardless of the normalization used, once again, $T^{*}$ is the temperature below which a strong temperature dependence emerges. The data further suggest that a very high energy scale is involved in the spectral weight redistribution associated with pseudogap opening. In addition, as energy-integrated spectral weight is conserved at a fixed momentum ${ }^{14}$, a change in the total spectral weight over a wide energy window in the occupied states at $k_{\mathrm{F}}$ could involve spectral weight redistribution from the unoccupied states, which often occurs with the breaking of particle-hole symmetry. This is consistent with the particle-hole symmetry breaking observed in the momentum structure (Fig. 1n). All of the above observations can be robustly reproduced and no sample surface degeneration was found during the measurement (Supplementary Figs S4-S6).

The opening of a pseudogap below $T^{*}$ that breaks the particlehole symmetry is accompanied by several anomalous temperaturedependent behaviours. When considering the spectral line shape evolution with temperature on the basis of a simple textbook picture for a metal, one expects a broadening of the spectra with increasing temperature because of the increased phase space available for inelastic electron scattering. Furthermore, the opening 
of a superconducting energy gap at low temperatures generally sharpens quasi particles by suppressing inelastic electron scattering. In stark contrast, the observed spectral evolution below $T^{*}$ goes to the opposite direction, suggesting that an unusual broadening mechanism is at work in the pseudogap state. In addition, the spectra no longer retain a Lorentzian line shape below $T^{*}$. Our data also provide important information missed in earlier studies. The pseudogap has previously been reported to disappear in a subtle manner, manifested as a filling-in of the gapped states in the vicinity of $E_{\mathrm{F}}\left(\mathrm{a}\right.$ few $\left.k_{\mathrm{B}} T\right)$ rather than a clear gap closing ${ }^{9-11}$. Different from those previous reports, our results uncover the pseudogap closing with a clearly shifting energy scale (Fig. 2c) and a concurrent spectral weight redistribution over a wide energy range (Fig. 2d).

Our observations collectively reveal a rich physical picture of the pseudogap phenomena beyond a simple extension of superconductivity that requires other ingredients in a complete picture. This picture is overall consistent and aligned with ref. 10, but differs in critical areas such as the assumption of particle-hole symmetry near the antinode. Our revelation of the broken-symmetry nature of the pseudogap rationalizes the competing relationship between the pseudogap state and superconductivity. Motivated by observations ${ }^{2-19,22-25}$ as well as theoretical implications ${ }^{26}$ of spatial symmetry breaking (densitywave-like correlations), we explore whether a density-wave picture can account for the observed change of band dispersion. We first consider a simple mode ${ }^{27}$ of long-range density-wave orders in the weak-coupling limit. Figure 3 shows the expected ARPES spectra from a calculation with the incommensurate checkerboard density-wave order of orthogonal wave vectors, [0.26 $\pi, 0]$ and $[0,0.26 \pi]$ (Fig. $3 \mathrm{a}$ ) and the commensurate $[\pi, \pi]$ density-wave order (Fig. 3b) for three different order parameters $V=15,30$ and $60 \mathrm{meV}$ (weighted blue curves from top to bottom, respectively). We find that the modelling can qualitatively reproduce some key aspects of our findings. Most importantly, there is no dispersion anomaly at $k_{\mathrm{F}}$ because the density-wave gap opens owing to band folding in a particle-hole asymmetric manner, which is very different from the superconducting gap. Rather, there exist back-bendings (arrows) markedly away from $k_{\mathrm{F}}$ (red dashed lines). Besides, these models provide a qualitatively better account for the pronounced shifting energy scale with temperature than the existence of homogeneous superconductivity. The most striking finding is the strong downward shift of the band bottom energy (note the similarity between Figs 1n and 3a), which hardly happens for superconductivity. The modelling, on the other hand, fails to describe the counterintuitive broadening of spectra with decreasing temperature observed in the present study. A model assuming a short-range nature (10 lattice constants) of the $[\pi, \pi]$ densitywave order ${ }^{28,29}$ can do a better job regarding the broadness of spectra in the gapped state (Fig. 3c). In this model, interestingly, a shorter density-wave correlation length is required to reproduce the broader spectra at lower temperatures. However, it is difficult to explain the wide energy range of spectral weight redistribution in the pseudogap state given the relatively low $T^{*}$ within this model. This suggests that the textbook starting point assuming extended Bloch waves is no longer accurate ${ }^{8}$. An unconventional explanation that captures the merits of these simple models for the order responsible for the pseudogap with emphasis on the effects of strong coupling thus seems to be necessary for our observations of the pseudogap. In particular, the anomalous broadness of the spectra points to the highly localized nature of this order.

Reminiscent of our ARPES observations, a particular form of broken spatial and particle-hole symmetry spectra is observed by momentum-integrated STM in the pseudogap phase at low temperature ${ }^{2,3,30}$. The observed nanoscale inhomogeneity associated with local density-wave order and dominated by high-energy states away from $E_{\mathrm{F}}$ is formally consistent with our spatially averaged ARPES observation, both pointing to the broken-symmetry nature and high-energy relevance of the pseudogap phase. The combined energy and momentum resolution of ARPES allows us to directly reveal particle-hole symmetry breaking not only in the spectral weight as examined in STM experiments but also in the momentum structure of the gap in the pseudogap state. Furthermore, by working in a much more extended temperature window, our ARPES study establishes: (1) the strikingly simple electronic structure of the true normal state above $T^{*}$ - a critical baseline to understand the pseudogap state that has not been experimentally established; and (2) a direct connection of the broken-symmetry state below $T^{*}$ with the pseudogap opening. As such, our finding depicts an integrated picture for an improved understanding of the pseudogap as a strong-coupling form of broken-symmetry state that emerges from a simple normal state above $T^{*}$ and most probably competes with superconductivity.

Received 25 May 2009; accepted 26 February 2010; published online 4 April 2010

\section{References}

1. Timusk, T. \& Statt, B. The pseudogap in high-temperature superconductors: An experimental survey. Rep. Prog. Phys. 62, 61-122 (1999).

2. Kohsaka, Y. et al. An intrinsic bond-centred electronic glass with unidirectional domains in underdoped cuprates. Science 315, 1380-1385 (2007).

3. Kohsaka, Y. et al. How Cooper pairs vanish approaching the Mott insulator in $\mathrm{Bi}_{2} \mathrm{Sr}_{2} \mathrm{CaCu}_{2} \mathrm{O}_{8+\delta}$. Nature 454, 1072-1078 (2008).

4. Hoffman, J. E. et al. A four unit cell periodic pattern of quasi-particle states surrounding vortex cores in $\mathrm{Bi}_{2} \mathrm{Sr}_{2} \mathrm{CaCu}_{2} \mathrm{O}_{8+\delta}$. Science 295, 466-469 (2002).

5. Vershinin, M. et al. Local ordering in the pseudogap state of the high- $T_{\mathrm{c}}$ superconductor $\mathrm{Bi}_{2} \mathrm{Sr}_{2} \mathrm{CaCu}_{2} \mathrm{O}_{8+\delta}$. Science 303, 1995-1998 (2004).

6. Shen, K. M. et al. Nodal quasiparticles and antinodal charge ordering in $\mathrm{Ca}_{2-x} \mathrm{Na}_{x} \mathrm{CuO}_{2} \mathrm{Cl}_{2}$. Science 307, 901-904 (2005).

7. Wise, W. D. et al. Charge-density-wave origin of cuprate checkerboard visualized by scanning tunnelling microscopy. Nature Phys. 4, 696-699 (2008).

8. Wise, W. D. et al. Imaging nanoscale Fermi-surface variations in an inhomogeneous superconductor. Nature Phys. 5, 213-216 (2009).

9. Ma, J.-H. et al. Coexistence of competing orders with two energy gaps in real and momentum space in the high temperature superconductor $\mathrm{Bi}_{2} \mathrm{Sr}_{2-x} \mathrm{La}_{x} \mathrm{CuO}_{6+\delta}$. Phys. Rev. Lett. 101, 207002 (2008)

10. Kondo, T., Khasanov, R., Takeuchi, T., Schmalian, J. \& Kaminski, A. Competition between the pseudogap and superconductivity in the high- $T_{c}$ copper oxides. Nature 457, 296-300 (2009).

11. Nakayama, K. et al. Evolution of a pairing-induced pseudogap from the superconducting gap of $(\mathrm{Bi}, \mathrm{Pb})_{2} \mathrm{Sr}_{2} \mathrm{CuO}_{6}$. Phys. Rev. Lett. 103, 047002 (2009).

12. Kanigel, A. et al. Evidence for pairing above the transition temperature of cuprate superconductors from the electronic dispersion in the pseudogap phase. Phys. Rev. Lett. 101, 137002 (2008).

13. Yang, H.-B. et al. Emergence of preformed Cooper pairs from the doped Mott insulating state in $\mathrm{Bi}_{2} \mathrm{Sr}_{2} \mathrm{CaCu}_{2} \mathrm{O}_{8+\delta}$. Nature 456, 77-80 (2008).

14. Randeria, M. et al. Momentum distribution sum rule for angle-resolved photoemission. Phys. Rev. Lett. 74, 4951-4954 (1995).

15. Wei, J. et al. Superconducting coherence peak in the electronic excitations of a single-layer $\mathrm{Bi}_{2} \mathrm{Sr}_{1.6} \mathrm{La}_{0.4} \mathrm{CuO}_{6+\delta}$ cuprate superconductor. Phys. Rev. Lett. 101, 097005 (2008)

16. Lee, W. S. et al. Abrupt onset of a second energy gap at the superconducting transition of underdoped Bi2212. Nature 450, 81-84 (2007).

17. Tanaka, K. et al. Distinct Fermi-momentum-dependent energy gaps in deeply underdoped Bi2212. Science 314, 1910-1913 (2006).

18. He, R.-H. et al. Energy gaps in the failed high- $T_{\mathrm{c}}$ superconductor $\mathrm{La}_{1.875} \mathrm{Ba}_{0.125} \mathrm{CuO}_{4}$. Nature Phys. 5, 119-123 (2009).

19. Baumberger, F., Greber, T. \& Osterwalder, J. Fermi surfaces of the two-dimensional surface states on vicinal $\mathrm{Cu}(111)$. Phys. Rev. B 64, 195411 (2001).

20. Ono, S. \& Ando, Y. Evolution of the resistivity anisotropy in $\mathrm{Bi}_{2} \mathrm{Sr}_{2-x} \mathrm{La}_{x} \mathrm{CuO}_{6+\delta}$ single crystals for a wide range of hole doping. Phys. Rev. B 67, 104512 (2003).

21. Zheng, G., Kuhns, P. L., Reyes, A. P., Liang, B. \& Lin, C. T. Critical point and the nature of the pseudogap of single-layered copper-oxide $\mathrm{Bi}_{2} \mathrm{Sr}_{2-x} \mathrm{La}_{x} \mathrm{CuO}_{6+\delta}$ superconductors. Phys. Rev. Lett. 94, 047006 (2005).

22. Tranquada, J. M., Sternlieb, B. J., Axe, J. D., Nakayama, Y. \& Uchida, S. Evidence for stripe correlations of spins and holes in copper oxide superconductors. Nature 375, 561-563 (1995).

23. Abbamonte, P. et al. Spatially modulated 'Mottness' in $\mathrm{La}_{2-x} \mathrm{Ba}_{x} \mathrm{CuO}_{4}$. Nature Phys. 1, 155-158 (2005).

24. Fujita, M., Goka, H., Yamada, K., Tranquada, J. M. \& Regnault, L. P. Stripe order, depinning, and fluctuations in $\mathrm{La}_{1.875} \mathrm{Ba}_{0.125} \mathrm{CuO}_{4}$ and $\mathrm{La}_{1.875} \mathrm{Ba}_{0.075} \mathrm{Sr}_{0.050} \mathrm{CuO}_{4}$. Phys. Rev. B 70, 104517 (2004). 
25. Doiron-Leyrau, N. et al. Quantum oscillations and the Fermi surface in an underdoped high- $T_{\mathrm{c}}$ superconductor. Nature 447, 565-568 (2007).

26. Chakravarty, S., Laughlin, R. B., Morr, D. K. \& Nayak, C. Hidden order in the cuprates. Phys. Rev. B 63, 094503 (2001).

27. Brouet, V. et al. Angle-resolved photoemission study of the evolution of band structure and charge density wave properties in $\mathrm{RTe}_{3}(\mathrm{R}=\mathrm{Y}, \mathrm{La}, \mathrm{Ce}, \mathrm{Sm}, \mathrm{Gd}$, Tb, and Dy). Phys. Rev. B 77, 235104 (2008).

28. Lee, P. A., Rice, T. M. \& Anderson, P. W. Fluctuation effects at a Peierls transition. Phys. Rev. Lett. 31, 462 (1973).

29. Grilli, M., Seibold, G., Di Ciolo, A. \& Lorenzana, J. Fermi surface dichotomy on systems with fluctuating order. Phys. Rev. B 79, 125111 (2009).

30. Johnston, S., Vernay, F. \& Devereaux, T. P. Impact of an oxygen dopant in $\mathrm{Bi}_{2} \mathrm{Sr}_{2} \mathrm{CaCu}_{2} \mathrm{O}_{8+\delta}$. Europhys. Phys. Lett. 86, 37007 (2009).

\section{Acknowledgements}

We thank W.-S. Lee, E. Berg, K. K. Gomes, B. Moritz, S. A. Kivelson, M. Grilli, H. Q. Lin

N. Nagaosa, A. Fujimori and J. Zaanen for helpful discussions and Y. Li for experimental assistance on SQUID measurements. R.-H.H. thanks the SGF for financial support. This work is supported by the Department of Energy, Office of Basic Energy Science under contract DE-AC02-76SF00515.

\section{Author contributions}

ARPES measurements were done by M.H., R.-H.H., K.T. and W.M. Y.Y. and H.E. grew and prepared the samples. M.H. and R.-H.H. analysed the ARPES data and wrote the paper with suggestions and comments by J.-P.T., H.Y, T.P.D. and Z.-X.S. ARPES simulations were done by M.H., R.-H.H., J.-P.T., H.Y. and T.P.D. R.G.M. and D.L. maintained the ARPES endstation. Z.H. and Z.-X.S. are responsible for project direction, planning and infrastructure.

\section{Additional information}

The authors declare no competing financial interests. Supplementary information accompanies this paper on www.nature.com/naturephysics. Reprints and permissions information is available online at http://npg.nature.com/reprintsandpermissions.

Correspondence and requests for materials should be addressed to Z.-X.S. 